\title{
Mathematical Modeling and Optimal Control Analysis on Sedentary Behavior and Physical Activity in Relation to Cardiovascular Disease (CVD)
}

\author{
Lawal Jibril ${ }^{1,}$, , Opeyemi Odetunde ${ }^{2}$ \\ ${ }^{1}$ Department of Mathematical Sciences, Faculty of Science, Federal University Gusau, Gusau, Nigeria \\ ${ }^{2}$ Department of Mathematics, Faculty of Physical Science, University of Ilorin, Ilorin, Nigeria
}

Email address:

ljibrail@gmail.com(L. Jibril), odetunde.o@unilorin.edu.ng (O. Odetund)

${ }^{*}$ Corresponding author

\section{To cite this article:}

Lawal Jibril, Opeyemi Odetunde. Mathematical Modeling and Optimal Control Analysis on Sedentary Behavior and Physical Activity in Relation to Cardiovascular Disease (CVD). Biomedical Statistics and Informatics. Vol. 5, No. 4, 2020, pp. 87-99.

doi: $10.11648 /$ j.bsi.20200504.13

Received: November 5, 2020; Accepted: November 21, 2020; Published: December 31, 2020

\begin{abstract}
Cardiovascular diseases (CVDs) have remained the leading causes of global death in the last 16 years which is the cause of mortality of 17.7 million people every year. Nowadays, people live in a time where sitting takes up the majority of their daily affairs. The sedentary behavior for prolonged periods of time can leads to a problem of deadly disease such as heart disease, obesity, and diabetes. In this paper a deterministic model for the effects of prolonged sitting is designed. The model, which consists of three ordinary differentials equations is developed and analyzed to study the optimal control analysis on sedentary behavior, physical activity in relation to cardiovascular disease (CVD) in a community. The solutions of the model uniquely exist, nonnegative for all $\mathrm{t} \geq 0$ with nonnegative initial conditions in $\mathrm{R}^{3}$, and bounded in a region $\Omega_{\mathrm{N}}$. The basic reproduction number which measures the relationship threshold is presented. The model was extended and optimal control theory was applied to examine optimal strategies for controlling or eradicating the new cases of CVD that may be borne due to a life of inactivity. The control measures comprises of education or sensitization $\mathrm{u}_{1}$, living a healthy lifestyle (good nutrition, weight management) $\mathrm{u}_{2}$, and getting plenty of physical activity $\mathrm{u}_{3}$. The impact of using possible combinations of the three intervention strategies was investigated and analyzed. The results of the optimal control model using Pontryagin maximum principle (PMP) revealed that combination of education or sensitization with any other control strategy yields better result to reduce or eradicate the risk of new cases of CVD from sedentary lifestyle.
\end{abstract}

Keywords: Deterministic Model, Physical Activity, Sedentary Behavior, Cardiovascular Disease, Optimal Control Analysis

\section{Introduction}

Over the last hundred years, there has been a large transformation from manual labor jobs for instance, farming, manufacturing, building to office jobs which is due to many contributing factors including globalization, outsourcing of jobs and technological advances more specifically internet and computers. In 1960, there was a general decline of jobs requiring moderate physical activity from $50 \%$ to $20 \%$, and one in two Americans had a physically demanding job, while in 2011 this ratio was one in five [16].

Modern lifestyle in nowadays requires working machines, which do much of the people's work, eliminating the need for manual labor. Working in the office by using those machines force people to sit there for eight or more hours a day. The recent studies shows that the average person spends more than half of his/her waking hours in an inactive state [5]. Epidemiological evidence suggests that sedentary behavior (loosely defined as sitting, television viewing, and couch time) is associated with increased risk for at least 35 chronic diseases/clinical conditions and increased mortality rates. Epidemiological reports also suggest that regular defined bouts of exercise may not protect against early mortality in certain populations if excessive sedentary behavior occurs 
over time [14], thus, making comparisons that sitting is the new smoking. Effects of a sedentary work life or lifestyle can be either direct or indirect. One of the most prominent direct effects of a sedentary lifestyle is an increased body mass index leading to obesity. A lack of physical activity is one of the leading causes of preventable disease worldwide [15].

Cardiovascular, or circulatory system supplies the body with blood. It consists of the heart, arteries, veins, and capillaries. Cardiovascular disease $(C V D)$ is a general term for conditions affecting the heart or blood vessels. It's usually associated with a build-up of fatty deposits inside the arteries [11]. Recently, CVDs are placed the number one cause of death globally. It was reported that more people die annually from CVDs than from any other cause [2]. There is now strong evidence that too much sitting is associated with an increased risk of developing cardiovascular disease and type 2 diabetes and for premature death from all-causes and cardiovascular disease [4].

An estimated 17.9 million people died from $C V D s$ in 2016 , representing $31 \%$ of all global deaths, of these deaths $85 \%$ are due to heart attack and stroke. Over three quarters of CVD deaths take place in low- and middle-income countries. Most cardiovascular diseases can be prevented by addressing behavioral risk factors such as hypertension, diabetes, tobacco use, unhealthy diet, obesity, physical inactivity, harmful use of alcohol and hyperlipidemia or already established disease. People with cardiovascular disease or who are at high cardiovascular risk need early detection and management using counselling and medicines, as appropriate $[2,3,13,17]$.

The coronary artery disease begins in the teenage years' and it is evident that plaques are formed in most people. Preventive measures instituted early are thought to have greater lifetime benefits. Healthy lifestyles will delay the progression of the disease, and there is hope that these diseases can be regressed before it causes $C V D$. Typical warning signs are chest pain, shortness of breath, palpitations and fatigue. Some consequences attached to $C V D$ indicated that there are at least 300,000 premature deaths and $\$ 90$ billion indirect healthcare costs are caused by obesity and sedentary lifestyle per year in the US alone. The risk is higher among those that sit still more than 4 hours per day and have $40 \%$ higher risk than those that sit fewer than 4 hours per day. However, those that exercise at least 4 hours per week are as healthy as those that sit fewer than 4 hours per day [1].

Deterministic epidemiology has started in the early twentieth century. Kermack and McKendrickk [18] work which was published in 1927 laid out a foundation for modeling infectious diseases, where all members of the population are assumed to be initially equally susceptible to the disease and confer complete immunity only after recovery.

The main objective of this paper is to develop a basic model of sedentary behavior and physical activity in relation to cardiovascular disease using compartmental model and apply Pontryagin maximum principle to study the model optimal control analysis to understand the deep dynamical nature of the relationship.

\section{Formulation of Sedentary Lifestyles Basic Model}

Kraus [8], a preventive cardiologist and research scientist at Duke University showed that both the risk of heart disease and risk factors for heart disease are strongly linked to family history. If there are family history of $C V D$, the risk of developing it increased. It was considered to have a family history of $C V D$ if either: a father or brother were diagnosed with $C V D$ before they were 55 and a mother or sister were diagnosed with CVD before they were 65 .

The sedentary behavior model is based on examining the dynamics of the sub-population of physical activity, $P A(t)$; Sedentary behavior, $S B(t)$ and Cardiovascular disease population, $C D(t)$; for convenience let $P A(t)=S(t)$, $S B(t)=I(t)$, and $C D(t)=C(t)$. Thus, the total population size at time $t$ is given by $N(t)=S(t)+I(t)+C(t)$.

Class of physical activity is increased due to human daily activities or potential contender's recruitment into physical activity class at a rate $\pi$. The physical activity population is reduced due to involvement in excessive sitting as a results of lack of information about danger that might lead to acquire cardiovascular disease or as a results of a family history of $C V D$ which was believed as a strong risk factor linked to the disease, at the rate $\beta$ and $\alpha$ respectively. Furthermore, it is assumed that the population of physical activity would rise due to encouraging people to be physically active and adopting physical activity behavior at a rate $\sigma$. Similarly, it is assumed that population of cardiovascular is reduced due to early detection of the symptoms for $C V D$ and immediately adopt changing of lifestyles and medication take place at a rate $\gamma$.

The class of sedentary population is increased due to change in lifestyle i.e. engaged in physical inactivity with a force of infection of the form $\beta S I$. At a chronic stage individual might progressed to $C V D$ class at a rate $\tau$ and increasing the population of $C V D$ as well. It is assumed that all classes suffer from natural death at a rate $\mu$ and there is $C V D$ induced death rate $\delta$.

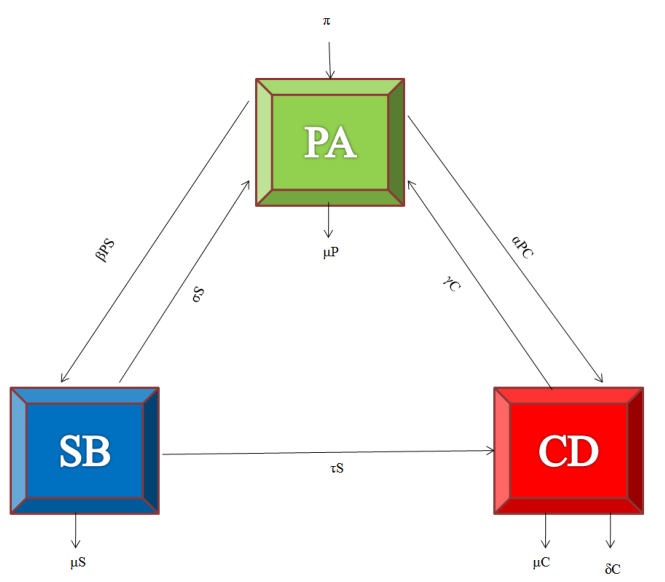

Figure 1. Compartmental Model diagram. 


\subsection{Assumptions of the Proposed Basic Model}

The study models the dynamics of sedentary behavior under the following assumptions:

1. the population mixes homogeneously.

2. Recruitments into population includes individuals that spent below seven hours in sitting.

3. It is assumed that individuals sitting for more than 9 hours increases the risk for being sedentary.

4. The model does not consider recovery class.

5. it is assumed that individuals leave the physical activity class via the force of infection $\beta S I$ and $\alpha S C$.

6. All parameters used are assumed to be positive.

\subsection{Model Equations}

The dynamical physical activity is given by the following nonlinear system

$$
\begin{gathered}
\frac{d S(t)}{d t}=\pi+\sigma I(t)+\gamma C(t)-(\beta I(t)+\alpha C(t)) S(t)-\mu S(t) \\
\frac{d I(t)}{d t}=\beta I(t) S(t)-(\sigma+\tau+\mu) I(t) \\
\frac{d C(t)}{d t}=\alpha C(t) S(t)+\tau I(t)-(\gamma+\mu+\delta) C(t)
\end{gathered}
$$

Subject to the nonnegative initial conditions

$$
S(0) \geq 0, I(0) \geq 0, C(0) \geq 0
$$

The total population at time $t$, denoted by $N(t)$ is given by

$$
N(t)=S(t)+I(t)+C(t)
$$

Thus, the model equation (1-3) parameters and variables are described in Table 1 below.

Table 1. Description of the Parameters and Variables of the Basic Model

\begin{tabular}{ll}
\hline Parameter/Variable & Description \\
\hline$\pi$ & $\begin{array}{l}\text { Individuals with less sitting hours ( }<5 \text { hours) } \\
\text { Rate at which sedentary individuals move to } \\
\text { physical activity class } \\
\text { Rate at which CVD individuals move to physical } \\
\text { activity class due to healthy lifestyles adjustment }\end{array}$ \\
$\gamma$ & $\begin{array}{l}\text { Rate at which sedentary individuals move to } \\
\text { CVD class }\end{array}$ \\
$\tau$ & Effective contact rate due to CVD disease \\
$\alpha$ & Effective contact rate due to Sedentary behaviour \\
$\beta$ & Natural death rate in all classes \\
$\mu$ & $C V D$ induced death \\
$\delta$ & Physical activity population at time $t$. \\
$P A(t)$ or $S(t)$ & Sedentary Behaviour population at time $t$. \\
$S A(t)$ or $I(t)$ & Cardiovascular patient at risk population at time $t$ \\
$C D(t)$ or $C(t)$ &
\end{tabular}

\subsection{Mathematical Analysis}

\subsubsection{Basic Properties of the Model}

The model system describes the dynamic of the sedentary behavior and physical activity in relation to cardiovascular disease $(C V D)$.

Before analyzing the dynamics of the basic model, firstly we discuss the basic properties of the model system (1-3). However, the model monitors changes in the human population, therefore it is assumed that all model variables and parameters to be nonnegative for all $t \geq 0$.

\subsubsection{Positivity of Solutions}

The model system is dealing with human populations, it is assumed that all the variables and parameters of the model are non-negative. By adding up the equations of the model system (1-3) we obtain:

$$
\begin{gathered}
\frac{d N(t)}{d t}=\pi+\sigma I(t)+\gamma C(t)-(\beta I(t)+\alpha C(t)) S(t) \\
-\mu S(t)+\beta I(t) S(t)-(\sigma+\tau+\mu) I(t) \\
+\alpha C(t) S(t)+\tau I(t)-(\gamma+\mu+\delta) C(t) \\
=\pi-\mu S(t)-\mu I(t)-\mu C(t)-\sigma I(t)-\delta C(t) \\
=\pi-\mu(S(t)+I(t)+C(t))-\sigma I(t)-\delta C(t) \\
\frac{d N(t)}{d t}=\pi-\mu N(t)
\end{gathered}
$$

We claim the following results

Theorem 1. (Positivity)

The solution set $(S, I, C)$ of the sedentary epidemiological model system $(1-3)$ with non-negative initial conditions (4), remains non-negative for all $t>0$.

Proof. Given that the initial conditions $S(0), I(0), C(0)$ are non-negative. It is observed that from the first equation, we deduce that for all $t>0$.

$$
\frac{d S}{d t} \geq-(\beta I(t)+\alpha C(t)+\mu) S(t)
$$

Let, $k=(\beta I(t)+\alpha C(t)+\mu)$

Substituting in equation (7) to obtain

$$
\frac{d S}{d t} \geq-k S(t)
$$

Integrating and solving equation (8) to obtain

$$
\begin{aligned}
& \ln |S| \geq \int-k d t+C \\
& |S| \geq \ell^{\int-k d t \ell^{C}} \\
& \therefore S(t) \geq S_{0} \ell^{\int-k d t}
\end{aligned}
$$


Hence,

$$
S(t) \geq S_{0} \ell^{\int-(\beta I(t)+\alpha C(t)+\mu) d t} \geq 0
$$

Where, $S_{0}$ is the population of susceptible individuals at $t=0$. Similarly, the remaining two equations are obtained that $I(0), C(0) \geq 0$. Therefore, we establish that any solution of the sedentary model equation $(1-3)$ is such that $\{(S, I, C)\} \in R_{+}^{3}$

Theorem 2. (Boundedness)

All solution of model system (1-3) is bounded and remain in the region $\Omega_{N}$.

Where,

$$
\Omega_{N}=\left\{(S, I, C) \in R_{+}^{3}: 0 \leq(S(t)+I(t)+C(t)) \leq \frac{\pi}{\mu}\right\}
$$

Proof: From equation (5) the total human population. This implies that,

$$
\begin{gathered}
\frac{d N}{d t}=\frac{d S}{d t}+\frac{d I}{d t}+\frac{d C}{d t} \\
\frac{d N(t)}{d t}=\pi+\sigma I(t)+\gamma C(t)-(\beta I(t)+\alpha C(t)) S(t)- \\
\mu S(t)+\beta I(t) S(t)-(\sigma+\tau+\mu) I(t)+\alpha C(t) S(t) \\
+\tau I(t)-(\gamma+\mu+\delta) C(t)
\end{gathered}
$$

On simplification, (13) becomes

$$
\frac{d N(t)}{d t}=\pi-\mu N(t)
$$

By integration and simplifying

$$
N(t) \leq \frac{\pi}{\mu}+C \ell^{-\mu t}
$$

Which implies

$$
C=\left(N(t)-\frac{\pi}{\mu}\right) \ell^{-\mu t}
$$

At $t=0$

$$
\left(N(0)-\frac{\pi}{\mu}\right) \leq C
$$

Therefore, from (15)

$$
\begin{gathered}
N(t)=N_{0} \ell^{-\mu t}+\frac{\pi}{\mu}\left(1-\ell^{-\mu t}\right) \\
\text { Hence, } N(t) \rightarrow \frac{\pi}{\mu} \quad \text { as } t \rightarrow \infty \\
\text { Thus, } \lim _{t \rightarrow \infty} N(t) \leq \frac{\pi}{\mu} \\
\text { And, } 0 \leq N(t) \leq \frac{\pi}{\mu} \\
\Omega_{N}=\left\{(S, I, C) \in R_{+}^{3}: 0 \leq(S(t)+I(t)+C(t)) \leq \frac{\pi}{\mu}\right\}
\end{gathered}
$$

Then,

This indicates that the population is biologically feasible into the region $\Omega_{N}$. Therefore, the solution of the model system $(1-3)$ with the initial condition (4) is bounded in the invariant region, $\Omega_{N}$ for all $t>0$. Hence, the model is well posed.

\section{Optimal Control Analysis for the Sedentary Model}

\subsection{Model Description}

Control measures are mechanisms put in place to prevent the occurrence or to eradicate the existence of a particular situation. Thus, we seek to address the impact of control measures that can be taken to reduce the risk of cardiovascular diseases that may arise from sedentary lifestyle. This is necessary in order to minimize or rather eradicate the new cases of $C V D$ that may be borne due to a life of inactivity. It is pertinent to state here that the risk of $C V D$ does not majorly depend on sedentary lifestyle as highlighted from the body of text, but too much sitting and non-activity may contribute to developing new cases of $C V D$ as obtained from literature [10]. To check the impact of some control parameters on the rate of $C V D$, we incorporated three control measures on the lifestyle of a susceptible individual. The control measures are education or sensitization on how to reduce the chance of developing $C V D$ $\left(u_{1}\right)$, living a healthy lifestyle that incorporates good nutrition, weight management $\left(u_{2}\right)$ and getting plenty of physical activity $\left(u_{3}\right)$. These measures if properly implemented increase the chances of people not developing $C V D$ while also reducing the population of people already managing $C V D$. Thus, with these measures in place, more people remain healthy while the sedentary and cardiovascular classes are depleted. These measures are incorporated into the model to obtain the following system of ODE. 


$$
\begin{gathered}
\frac{d S(t)}{d t}=\pi+\sigma I(t)+\gamma C(t)-\left(1-u_{1}\right)(\beta I(t)+\alpha C(t)) S(t)-\mu S(t)+\left(u_{2}+u_{3}\right)(I+C) \\
\frac{d I(t)}{d t}=\left(1-u_{1}\right) \beta I(t) S(t)-(\sigma+\tau+\mu) I(t)-\left(u_{2}+u_{3}\right) I \\
\frac{d C(t)}{d t}=\left(1-u_{1}\right) \alpha C(t) S(t)+\tau I(t)-(\gamma+\mu+\delta) C(t)-\left(u_{2}+u_{3}\right) C
\end{gathered}
$$

Thus, the objectives are to: (i) minimize the occurrence of new $C V D$ as a result of sedentary lifestyle through the above mentioned controls strategies, (ii) manage/reduce the population of CVD patients using the aforementioned mechanisms of physical exercises in addition to the therapeutic treatments, and (iii) create proper awareness for the upcoming generation against the risk of sedentary lifestyle on their health. The objective functional together with the proposed optimal control problem for equations (23), (24) and (25) was derived as:

$$
J\left(u_{1}(t), u_{2}(t), u_{3}(t)\right)=\int_{t_{0}}^{t_{f}}\left(C_{1} S(t)-C_{2} I(t)-\frac{1}{2}\left[K_{1} u_{1}^{2}(t)+K_{2} u_{2}^{2}(t)+K_{3} u_{3}^{2}(t)\right]\right) d t
$$

Subject to state variables $S(t), I(t)$ and $C(t)$ as given in (23), (24) and (25) and $S(0)=S_{0}, I(0)=I_{0}, C(0)=C_{0}$.

Where $C_{1} S(t)$ denotes the benefits obtained from living a healthy lifestyle that incorporates good nutrition, weight management and getting plenty of physical activity, $C_{2} I(t)$ represent the effect of education and sensitization about the danger of sedentary lifestyle on the people that are prone to such a lifestyle so that new case(s) $C V D$ can be avoided, $K_{i} u_{i}^{2}$ are the systemic cost associated with each of the control measures. The constants $K_{1}, K_{2}, K_{3}$ denote the weighting constants attached to education, healthy lifestyle, regular physical exercises, weight management and good nutrition, $u_{1}, u_{2}, u_{3}$ are the control variables which are bounded and Lebesque integrable (measurable functions that are defined on a fixed interval and satisfies $0 \leq u_{i}(t) \leq 1$, for $i=1,2,3$.

The goal is to increase the awareness towards importance of the control measures so that more people will be engaged so that there will be no/fewer cases $C V D$ as a result of living a sedentary lifestyle. Hence, the optimal solution desired denoted as $u_{1}{ }^{*}, u_{2}{ }^{*}, u_{3}{ }^{*}$ maximizes the effectiveness of the control measures such that

$$
J\left(u_{1}{ }^{*}(t), u_{2}{ }^{*}(t), u_{3}{ }^{*}(t)\right)=\max _{u} J\left(u_{1}(t), u_{1}(t), u_{1}(t): u_{1}(t), u_{1}(t), u_{1}(t) \in \bigcup\right)
$$

And the set

$$
U=\left\{u_{1}(t), u_{2}(t), u_{3}(t) \in L^{1}\left(t_{0}, t_{f}\right)<1\right\}, \quad i=1,2,3 \text { is the }
$$

control set where $L^{1}\left(t_{0}, t_{f}\right)$ denote Lebesque integral of the functional equation (26). The state system (23), (24) and (25) together with the objective functional (26) shall be used to analyze the optimal control of the model.

\subsection{Pontryagins'Maximum Principle ( $P M P$ )}

We adopted the criteria for existence of optimal solution established by [12] as adopted and used by several authors including [9]. Pontryagins' Maximum Principle ( $P M P$ ) will be employed to determine the optimal control solution set for $u_{1}, u_{2}$ and $u_{3}$ with their associated conditions. The PMP changes (26), (23), (24) and (25) into a problem of minimizing pointwise a Hamiltonian $H$ with respect to $\left(u_{1}, u_{2}, u_{3}\right)$ simply as:

$$
H\left(S, I, C, u_{1}, u_{2}, u_{3}, \lambda_{i}\right)=f\left(S, I, C, u_{i}\right)+\lambda_{i} h\left(S, I, C, u_{i}\right)
$$

Where $f\left(S, I, C, u_{i}\right)$ denotes the integrand in (31), $\lambda_{i}$ is the adjoint (Lagrange multiplier) and $h\left(S, I, C, u_{i}\right)$ is the right hand side of the state system (28), (29) and (30).

Thus, the desired Hamiltonian $H$ or the objective functional was obtained as:

$$
\left.\begin{array}{l}
H\left(S, I, C, u_{1}, u_{2}, u_{3}, \lambda_{i}\right)=C_{1} S(t)-C_{2} I(t)-\frac{1}{2}\left[K_{1} u_{1}^{2}+K_{2} u_{2}^{2}+K_{3} u_{3}^{2}\right]+ \\
\lambda_{1}\left(\pi+\sigma I(t)+\gamma C(t)-\left(1-u_{1}\right)(\beta I(t)+\alpha C(t)) S(t)-\mu S(t)+\left(u_{2}+u_{3}\right)(I+C)\right)+ \\
\lambda_{2}\left(\left(1-u_{1}\right) \beta I(t) S(t)-(\sigma+\tau+\mu) I(t)-\left(u_{2}+u_{3}\right) I\right)+ \\
\lambda_{3}\left(\left(1-u_{1}\right) \alpha C(t) S(t)+\tau I(t)-(\gamma+\mu+\delta) C(t)-\left(u_{2}+u_{3}\right) C\right)
\end{array}\right\}
$$


Having formed the Hamiltonian, we want to test for the optimal control set $\left\{u_{1}{ }^{*}, u_{2}{ }^{*}, u_{3}{ }^{*}\right\}$ that minimizes the new cases of $C V D$ and the cost associated with $J\left(u_{1}, u_{2}, u_{3}\right)$ over the invariant region which can be obtained from:

i. Optimality condition: $\frac{\partial H}{\partial u_{i}}=0$ for $i=1,2,3$

ii. Adjoint functions $\frac{d \lambda_{i}}{d t}=-\frac{\partial H}{\partial x_{i}}$ for $i=1,2,3$ with transversality condition that $\lambda_{i}\left(t_{f}\right)=0$ where $x_{i}$ represent equations corresponding to the state variables.

Theorem 1: Suppose $u_{i}^{*}(t), i=1,2,3$ are optimal solutions together with the corresponding solutions $S^{*}, I^{*}, C^{*}$ for problem (26), then there exist a piecewise differentiable adjoint variable $\lambda_{i}(t)$ such that:

$$
\left.\begin{array}{l}
\frac{d \lambda_{1}}{d t}=\left(\lambda_{1}-\lambda_{2}\right) \beta I^{*}\left(1-u_{1}^{*}\right)+\left(\lambda_{1}-\lambda_{3}\right) \alpha C^{*}\left(1-u_{1}^{*}\right)+\mu \lambda_{1}-C_{1} \\
\frac{d \lambda_{2}}{d t}=\left(\lambda_{2}-\lambda_{1}\right)\left(-\beta S^{*}\left(1-u_{1}^{*}\right)+\sigma+u_{2}^{*}+u_{3}^{*}\right)+\left(\lambda_{2}-\lambda_{3}\right) \tau+\mu \lambda_{2}-C_{2} \\
\frac{d \lambda_{3}}{d t}=\left(\lambda_{3}-\lambda_{1}\right)\left(-\alpha S^{*}\left(1-u_{1}^{*}\right)+\gamma+u_{2}^{*}+u_{3}^{*}\right)+\lambda_{3}(\mu+\delta)
\end{array}\right\}
$$

Proof:

Recall the Hamiltonian $H$ in (27), it is sufficient to obtain the partial derivative of $H$ with respect to each of the state variables $S, I, C$. Since the adjoint condition established by PMP is given as $\frac{d \lambda_{i}}{d t}=-\frac{\partial H}{\partial x_{i}}$ for $i=1,2,3$. Thus,

$$
\begin{aligned}
\frac{d \lambda_{1}}{d t} & =-\frac{\partial H}{\partial S} \\
\frac{d \lambda_{2}}{d t} & =-\frac{\partial H}{\partial I} \\
\frac{d \lambda_{3}}{d t} & =-\frac{\partial H}{\partial C}
\end{aligned}
$$

Thus,

$$
\frac{\partial H}{\partial S}=C_{1}+\lambda_{1}\left(-\left(\left(1-u_{1}\right)(\beta I+\alpha C)+\mu\right)+\lambda_{2}(\beta I)\left(1-u_{1}\right)+\lambda_{3}(\alpha C)\left(1-u_{1}\right)\right)
$$

Applying the adjoint criteria on (29) at optimal solution to obtain

$$
\left.\begin{array}{l}
\frac{d \lambda_{1}}{d t}=-\frac{\partial H}{\partial S}=-C_{1}+\lambda_{1}\left(-\left(\left(1-u_{1}\right)(\beta I+\alpha C)+\mu\right)+\lambda_{2}(\beta I)\left(1-u_{1}\right)+\lambda_{3}(\alpha C)\left(1-u_{1}\right)\right) \\
\frac{d \lambda_{1}}{d t}=\left(\lambda_{1}-\lambda_{2}\right)\left(\beta I^{*}\right)\left(1-u_{1}^{*}\right)+\left(\lambda_{1}-\lambda_{3}\right)\left(\alpha C^{*}\right)\left(1-u_{1}^{*}\right)+\mu \lambda_{1}-C_{1}
\end{array}\right\}
$$

Similarly, obtaining the partial derivative of $H$ with respect to $I$ and $C$ and applying the adjoint criteria as above to obtain:

$$
\begin{aligned}
& \frac{d \lambda_{2}}{d t}=-\frac{\partial H}{\partial I}=-\left(-C_{2}+\lambda_{1}\left(\sigma-\beta S^{*}\left(1-u_{1}^{*}\right)+u_{2}^{*}+u_{3}^{*}\right)+\lambda_{2}\left(\beta S^{*}\left(1-u_{1}^{*}\right)-\sigma-\tau-\mu-u_{2}^{*}-u_{3}^{*}\right)+\lambda_{3}(\tau)\right) \\
& \frac{d \lambda_{2}}{d t}=\left(\lambda_{2}-\lambda_{1}\right)\left(-\beta S^{*}\left(1-u_{1}^{*}\right)+\sigma+u_{2}^{*}+u_{3}^{*}\right)+\left(\lambda_{2}-\lambda_{3}\right)(\tau)+\mu \lambda_{2}+C_{2}
\end{aligned}
$$

And

$$
\begin{aligned}
& \frac{d \lambda_{3}}{d t}=-\frac{\partial H}{\partial C}=-\left(\lambda_{1}\left(\gamma-\alpha S^{*}\left(1-u_{1}^{*}\right)+u_{2}^{*}+u_{3}^{*}\right)+\lambda_{3}\left(\alpha S^{*}-\gamma-\delta-\mu-u_{1}^{*}-u_{2}^{*}-u_{3}^{*}\right)\right) \\
& \frac{d \lambda_{3}}{d t}=\left(\lambda_{3}-\lambda_{1}\right)\left(-\alpha S^{*}\left(1-u_{1}^{*}\right)+\gamma+u_{2}^{*}+u_{3}^{*}\right)+(\mu+\delta) \lambda_{3}
\end{aligned}
$$


Thus, combining (30), (31) and (32) establishes the proof of the theorem.

Theorem 2: There exist an optimal solution set $\left\{u_{1}{ }^{*}, u_{2}{ }^{*}, u_{3}{ }^{*}\right\}$ that minimizes the functional $J\left(u_{1}, u_{2}, u_{3}\right)$ in the invariant region of solution and is given by:

$$
\begin{aligned}
& u_{1}^{*}=\max \left\{0, \min \left\{\frac{\left(\lambda_{1}-\lambda_{2}\right) \beta I^{*} S^{*}+\left(\lambda_{1}-\lambda_{3}\right) \alpha C^{*} S^{*}}{K_{1}}, 1\right\}\right\} \\
& u_{2}^{*}=\max \left\{0, \min \left\{\frac{\left(\lambda_{1}-\lambda_{2}\right) I^{*}+\left(\lambda_{1}-\lambda_{3}\right) C^{*}}{K_{2}}, 1\right\}\right\} \\
& u_{3}^{*}=\max \left\{0, \min \left\{\frac{\left(\lambda_{1}-\lambda_{2}\right) I^{*}+\left(\lambda_{1}-\lambda_{3}\right) C^{*}}{K_{3}}, 1\right\}\right\}
\end{aligned}
$$

Proof:

By the application of $P M P$ optimality condition on the Hamiltonian $H$ (partially differentiating $H$ with respect to each control variables), we have the following equations:

$$
\begin{aligned}
& \frac{\partial H}{\partial u_{1}}=-K_{1} u_{1}+\lambda_{1}(\beta I+\alpha C) S+\lambda_{2}(-\beta I S)+\lambda_{3}(-\alpha C S) \\
& \frac{\partial H}{\partial u_{1}}=0 \Rightarrow u_{1}=\frac{\left(\lambda_{1}-\lambda_{2}\right) \beta I S+\left(\lambda_{1}-\lambda_{3}\right) \alpha C S}{K_{1}}
\end{aligned}
$$

Similarly,

$$
\frac{\partial H}{\partial u_{2}}=-K_{2} u_{2}+\lambda_{1}(I+C)+\lambda_{2}(-I)+\lambda_{3}(-C)
$$

And

$$
\frac{\partial H}{\partial u_{3}}=-K_{3} u_{3}+\lambda_{1}(I+C)+\lambda_{2}(-I)+\lambda_{3}(-C)
$$

From where we obtained:

$$
\left.\begin{array}{l}
u_{2}=\frac{\left(\lambda_{1}-\lambda_{2}\right) I+\left(\lambda_{1}-\lambda_{3}\right) C}{K_{2}} \\
\text { and, } \\
u_{3}=\frac{\left(\lambda_{1}-\lambda_{2}\right) I+\left(\lambda_{1}-\lambda_{3}\right) C}{K_{3}}
\end{array}\right\}
$$

Combining equations (38) and (39) to obtain:

$$
\left.\begin{array}{l}
u_{1}=\frac{\left(\lambda_{1}-\lambda_{2}\right) \beta I S+\left(\lambda_{1}-\lambda_{3}\right) \alpha C S}{K_{1}} \\
u_{2}=\frac{\left(\lambda_{1}-\lambda_{2}\right) I+\left(\lambda_{1}-\lambda_{3}\right) C}{K_{2}} \\
u_{3}=\frac{\left(\lambda_{1}-\lambda_{2}\right) I+\left(\lambda_{1}-\lambda_{3}\right) C}{K_{3}}
\end{array}\right\}
$$

Characterization for optimal control is computed on the set $\cup=\left\{u_{1}(t), u_{2}(t), u_{3}(t) \in L^{1}\left(t_{0}, t_{f}\right)<1\right\}$ Such that at the optimal stage $t_{f}:\left\{t_{f}: 0<u_{i}^{*}\left(t_{f}\right)<1, \quad i=1,2,3\right\}$. Thus by using control space property by Lenhart [9] each control equation in (35) becomes:

$$
\begin{gathered}
u_{1}^{*}=\left\{\begin{array}{lr}
0, & \text { if } \frac{\left(\lambda_{1}-\lambda_{2}\right) \beta I^{*} S^{*}+\left(\lambda_{1}-\lambda_{3}\right) \alpha C^{*} S^{*}}{K_{1}} \leq 0 \\
\frac{\left(\lambda_{1}-\lambda_{2}\right) \beta I^{*} S^{*}+\left(\lambda_{1}-\lambda_{3}\right) \alpha C^{*} S^{*}}{K_{1}}, & \text { if } 0<\frac{\left(\lambda_{1}-\lambda_{2}\right) \beta I^{*} S^{*}+\left(\lambda_{1}-\lambda_{3}\right) \alpha C^{*} S^{*}}{K_{1}}<1 \\
1, & \text { if } \frac{\left(\lambda_{1}-\lambda_{2}\right) \beta I^{*} S^{*}+\left(\lambda_{1}-\lambda_{3}\right) \alpha C^{*} S^{*}}{K_{1}} \geq 1
\end{array}\right. \\
u_{2}^{*}=\left\{\begin{array}{lc}
\frac{\left(\lambda_{1}-\lambda_{2}\right) I^{*}+\left(\lambda_{1}-\lambda_{3}\right) C^{*}}{K_{2}}, & \text { if } 0<\frac{\left(\lambda_{1}-\lambda_{2}\right) I^{*}+\left(\lambda_{1}-\lambda_{3}\right) C^{*}}{K_{1}}<1 \\
1, & \text { if } \frac{\left(\lambda_{1}-\lambda_{2}\right) I^{*}+\left(\lambda_{1}-\lambda_{3}\right) C^{*}}{K_{1}} \geq 1
\end{array}\right.
\end{gathered}
$$




$$
u_{3}^{*}=\left\{\begin{array}{l}
0, \\
\frac{\left(\lambda_{1}-\lambda_{2}\right) I^{*}+\left(\lambda_{1}-\lambda_{3}\right) C^{*}}{K_{3}}, \\
1,
\end{array}\right.
$$

Each of equations (36), (37) and (38) can compactly be written as co-state equations at optimum condition and given as:

$$
\left.\begin{array}{l}
u_{1}^{*}=\max \left\{0, \min \left\{\frac{\left(\lambda_{1}-\lambda_{2}\right) \beta I^{*} S^{*}+\left(\lambda_{1}-\lambda_{3}\right) \alpha C^{*} S^{*}}{K_{1}}, 1\right\}\right\} \\
u_{2}^{*}=\max \left\{0, \min \left\{\frac{\left(\lambda_{1}-\lambda_{2}\right) I^{*}+\left(\lambda_{1}-\lambda_{3}\right) C^{*}}{K_{2}}, 1\right\}\right\} \\
u_{3}^{*}=\max \left\{0, \min \left\{\frac{\left(\lambda_{1}-\lambda_{2}\right) I^{*}+\left(\lambda_{1}-\lambda_{3}\right) C^{*}}{K_{3}}, 1\right\}\right\}
\end{array}\right\}
$$

This complete the proof of theorem 2 .

The co-state equations in (39) showed that each control has relatively the same impact as obtained from their respective

$$
\begin{aligned}
\text { if } \frac{\left(\lambda_{1}-\lambda_{2}\right) I^{*}+\left(\lambda_{1}-\lambda_{3}\right) C^{*}}{K_{3}} \leq 0 \\
\text { if } 0<\frac{\left(\lambda_{1}-\lambda_{2}\right) I^{*}+\left(\lambda_{1}-\lambda_{3}\right) C^{*}}{K_{3}}<1 \\
\text { if } \frac{\left(\lambda_{1}-\lambda_{2}\right) I^{*}+\left(\lambda_{1}-\lambda_{3}\right) C^{*}}{K_{3}} \geq 1
\end{aligned}
$$

equations, however, the determining factor is the weighting constant $K_{i}$ (or the cost associated with obtaining maximum benefits from each control).

\section{Numerical Simulation for the Optimal Control Problem}

The control profile for the state variables, adjoint equations and the co-state equations were numerically simulated using Runge-Kutta order 4 method. The initial conditions used were $S(0)=100, I(0)=30, C(0)=15$ and the parameters value were either assumed or obtained from literature cited in this work.

Table 2. Parameters and Estimated values for Sedentary Model.

\begin{tabular}{llll}
\hline Parameter & Description & Value & Source \\
\hline$\pi$ & less sitting hours $(<5$ hours) & 20 & Assumed \\
$\sigma$ & sedentary individuals move to physical activity class & 0.5 & Assumed \\
$\gamma$ & CVD individuals move to physical activity class & 0.2 & Assumed \\
$\tau$ & sedentary individuals move to CVD class & 0.15 & Assumed \\
$\alpha$ & contact rate due to CVD & 0.15 & Assumed \\
$\beta$ & contact rate due to SB & 0.75 & Assumed \\
$\mu$ & Natural death & 0.2 & {$[7]$} \\
$\delta$ & CVD induced death & 0.08 & Assumed \\
$u_{1}, u_{2}, u_{3}$ & Control variables & 0,1 & {$[6]$} \\
\hline
\end{tabular}

The graphs for both control model and non-controlled model were plotted together under several circumstances and were given below:
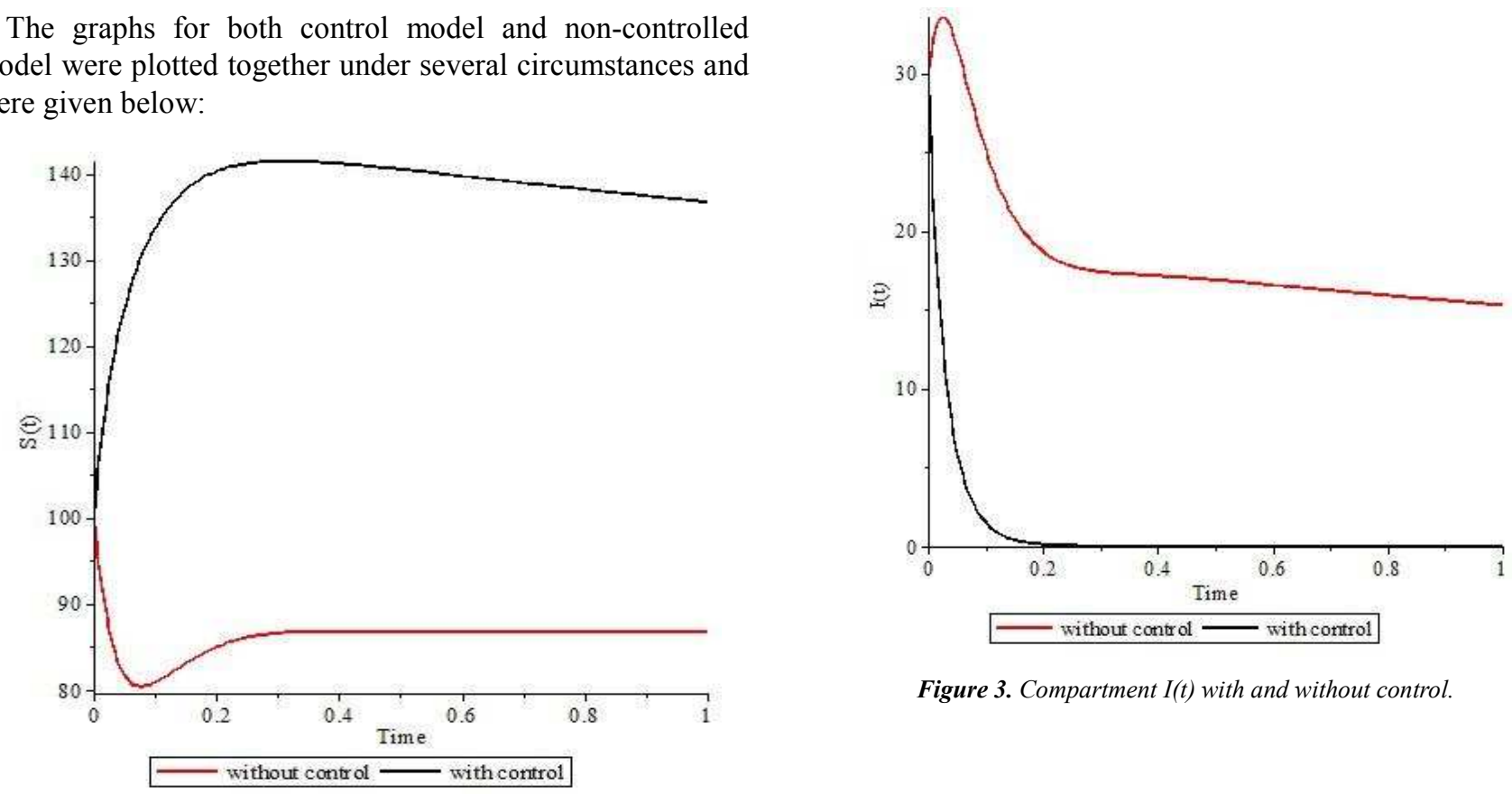

Figure 3. Compartment $I(t)$ with and without control.

Figure 2. Compartment $S(t)$ with and without control. 


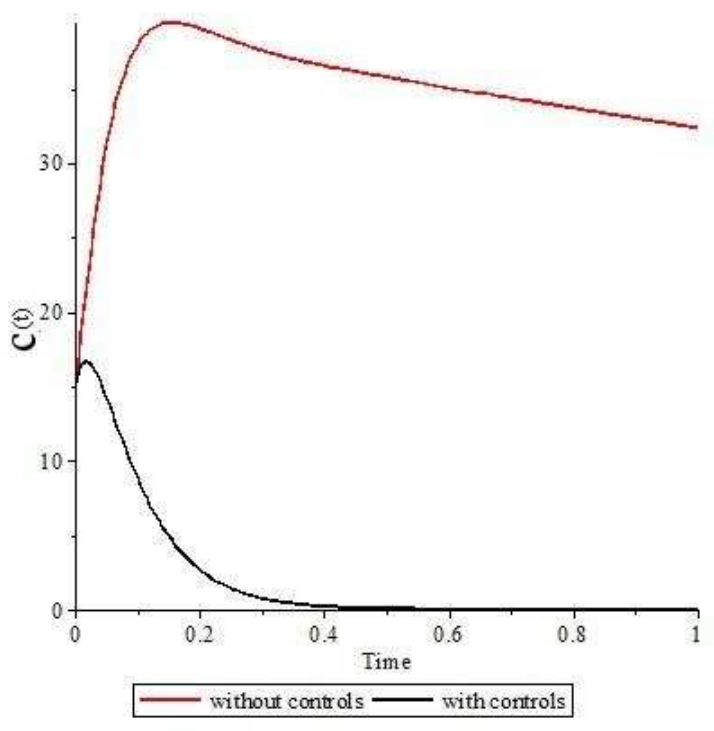

Figure 4. Compartment $I(t)$ with and without control.

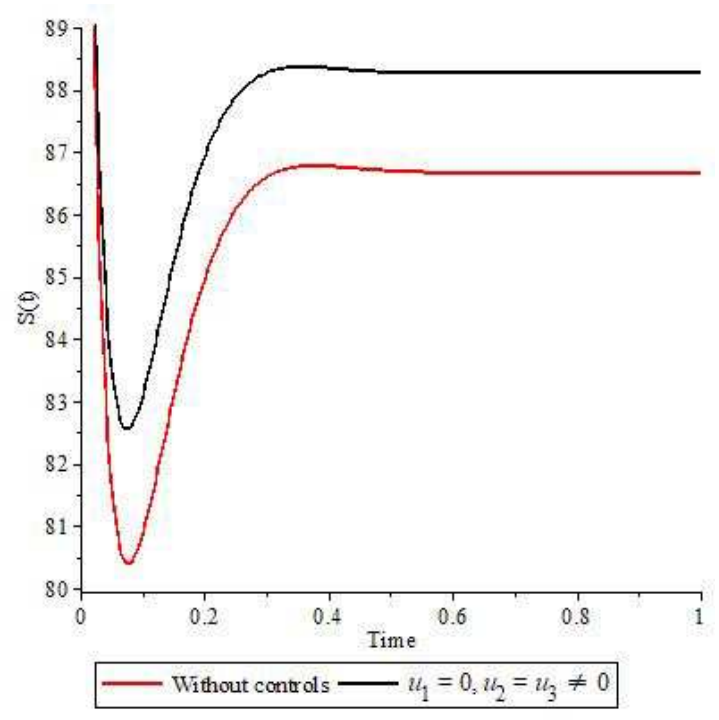

Figure 5. Compartment $S(t)$ with two controls, $u_{1}=0$.

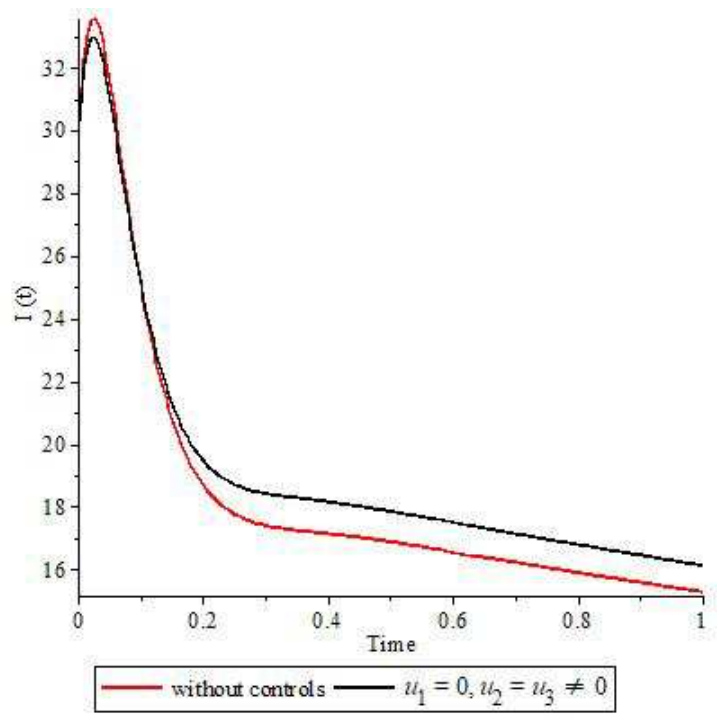

Figure 6. Compartment $I(t)$ with two controls, $u_{1}=0$.

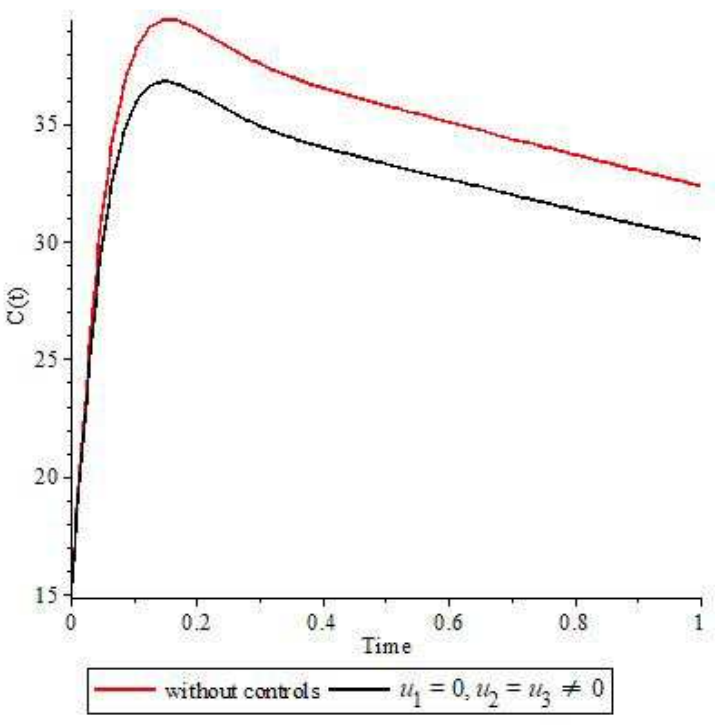

Figure 7. Compartment $C(t)$ with two controls, $u_{1}=0$.

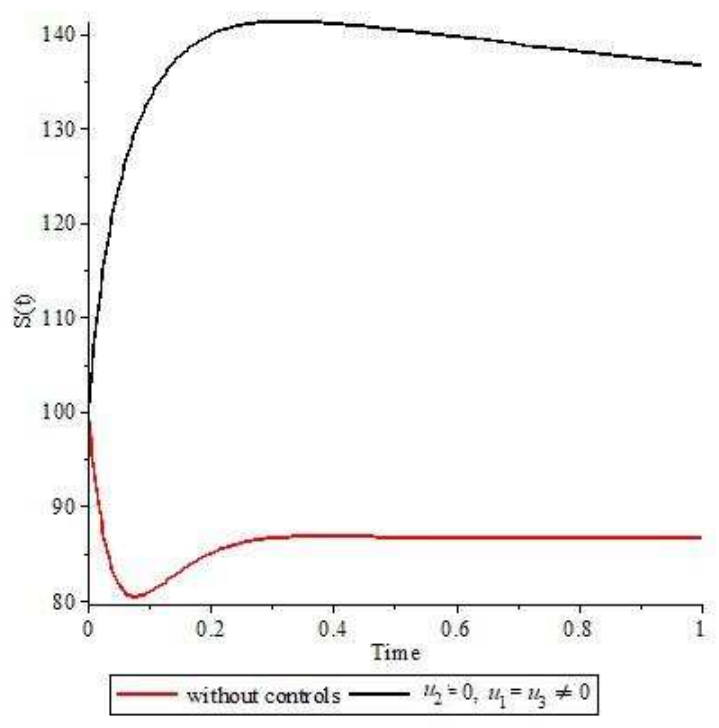

Figure 8. Compartment $S(t)$ with two controls, $u_{2}=0$.

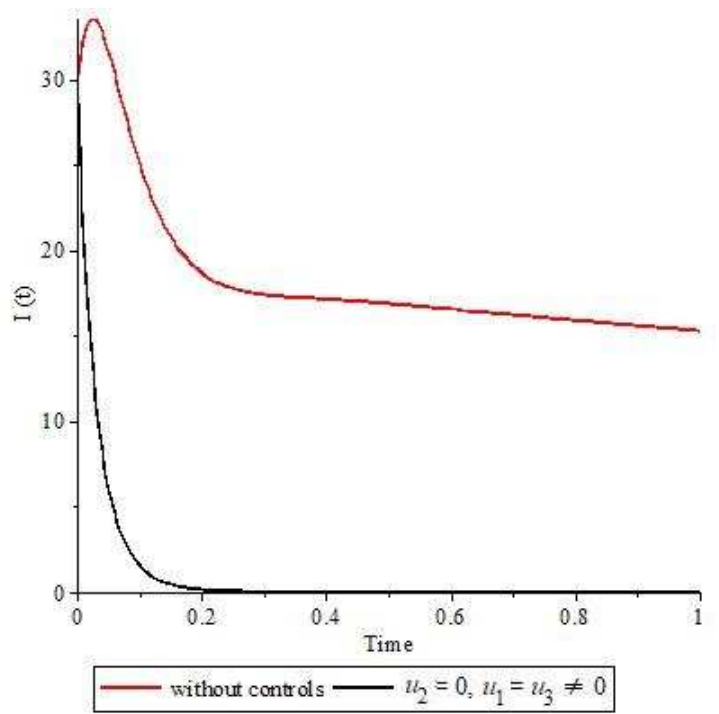

Figure 9. Compartment $I(t)$ with two controls, $u_{2}=0$. 


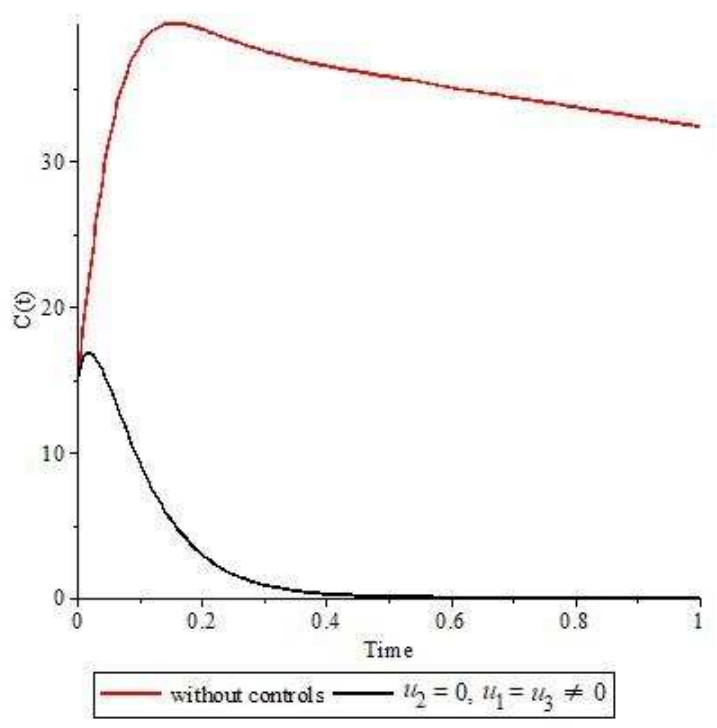

Figure 10. Compartment $C(t)$ with two controls, $u_{2}=0$.

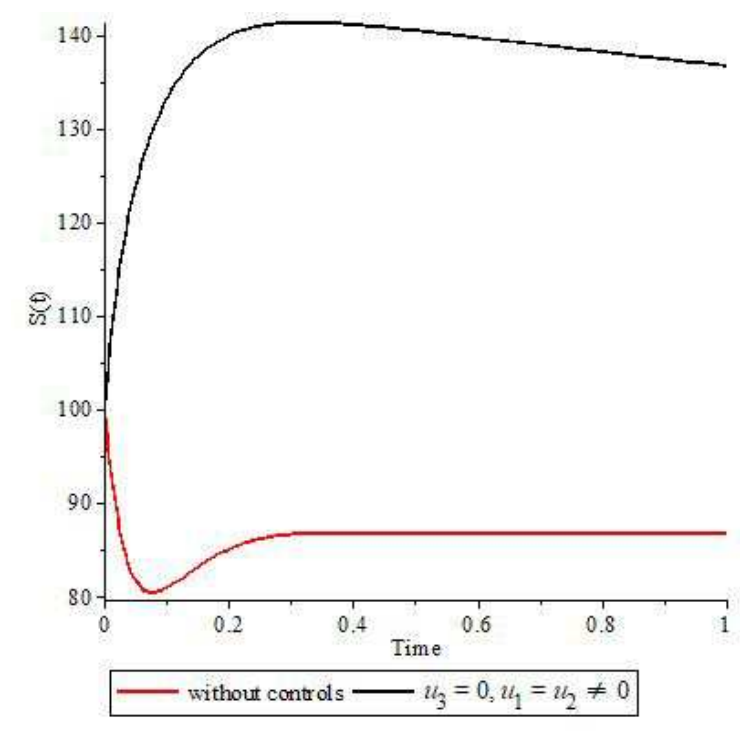

Figure 11. Compartment $S(t)$ with two controls, $u_{2}=0$.

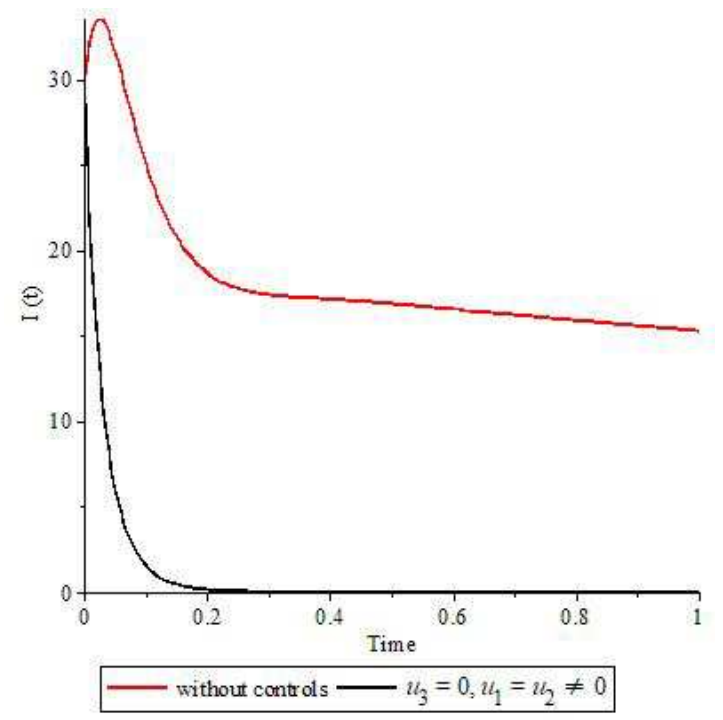

Figure 12. Compartment I (t) with two controls, $u_{2}=0$.

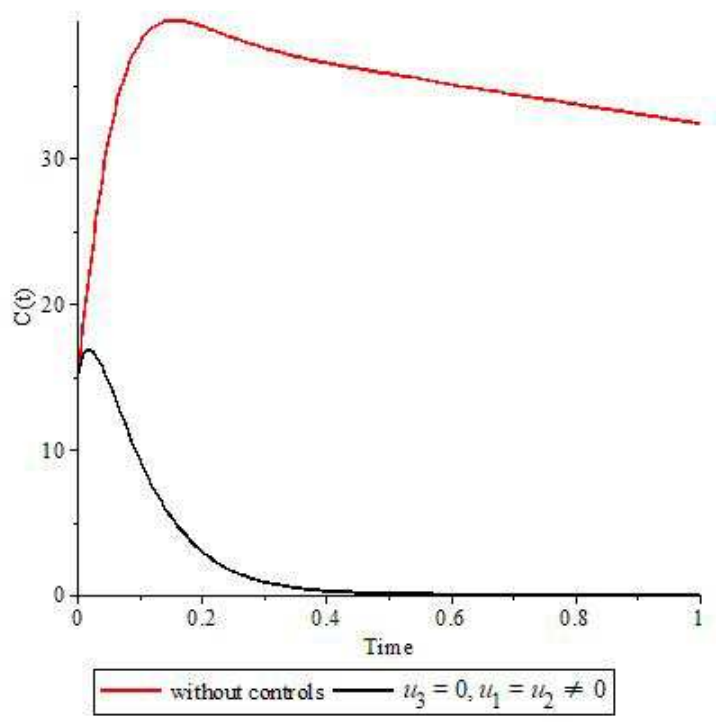

Figure 13. Compartment $C(t)$ with two controls, $u_{2}=0$.

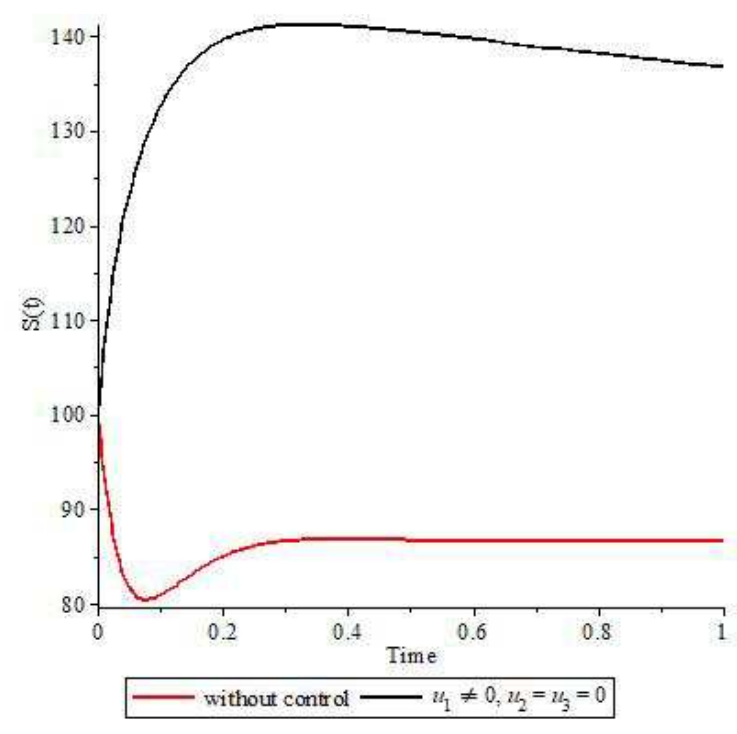

Figure 14. Compartment $S(t)$ with one controls, $u_{1} \neq 0$.

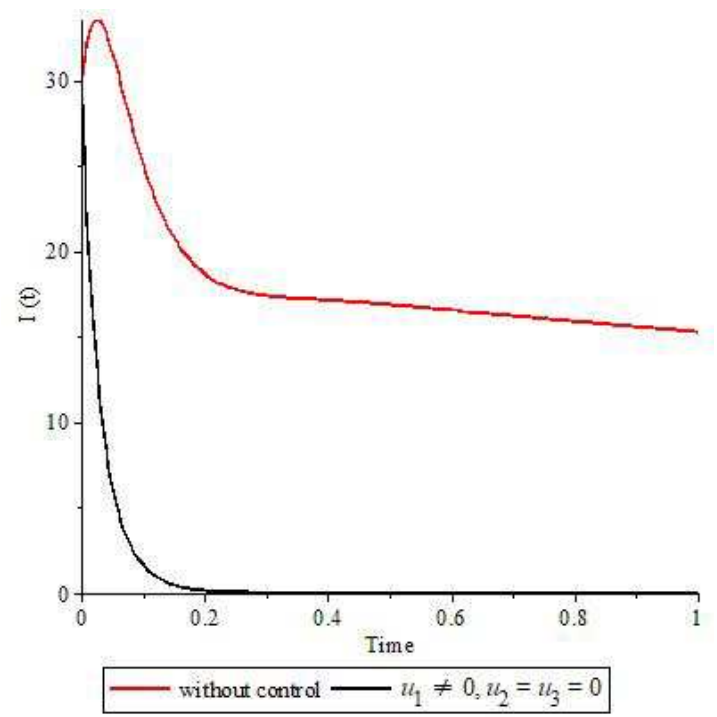

Figure 15. Compartment $I(t)$ with one controls, $u_{1} \neq 0$. 


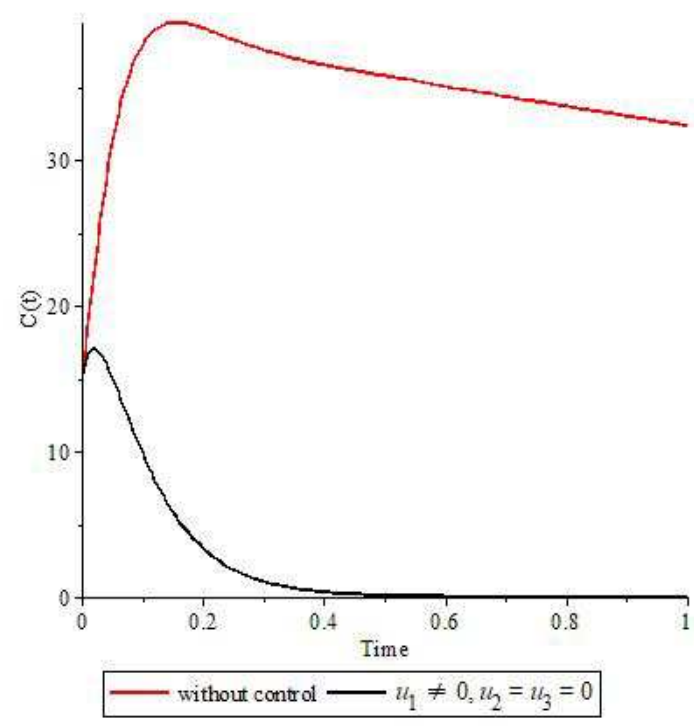

Figure 16. Compartment $C(t)$ with one controls, $u_{1} \neq 0$.

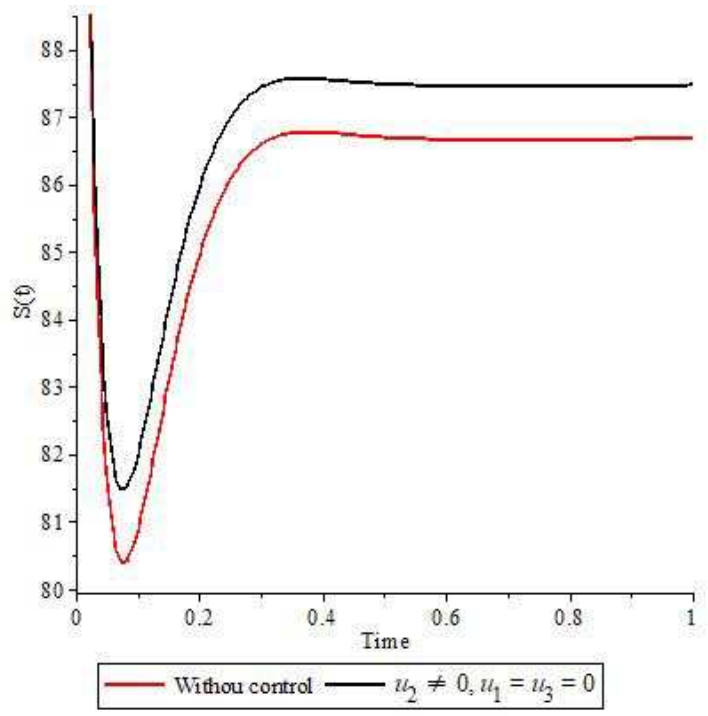

Figure 17. Compartment $S(t)$ with one controls, $u_{2} \neq 0$.

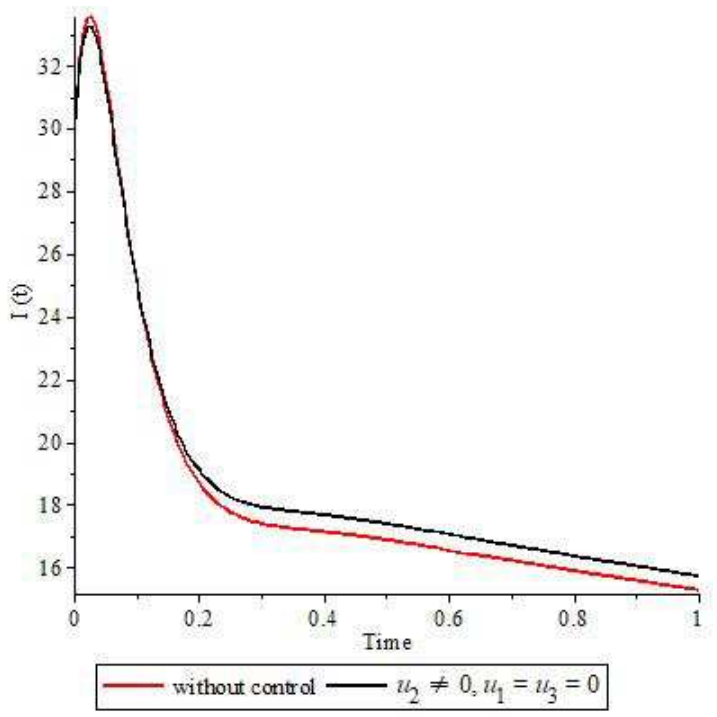

Figure 18. Compartment $I(t)$ with one controls, $u_{2} \neq 0$.

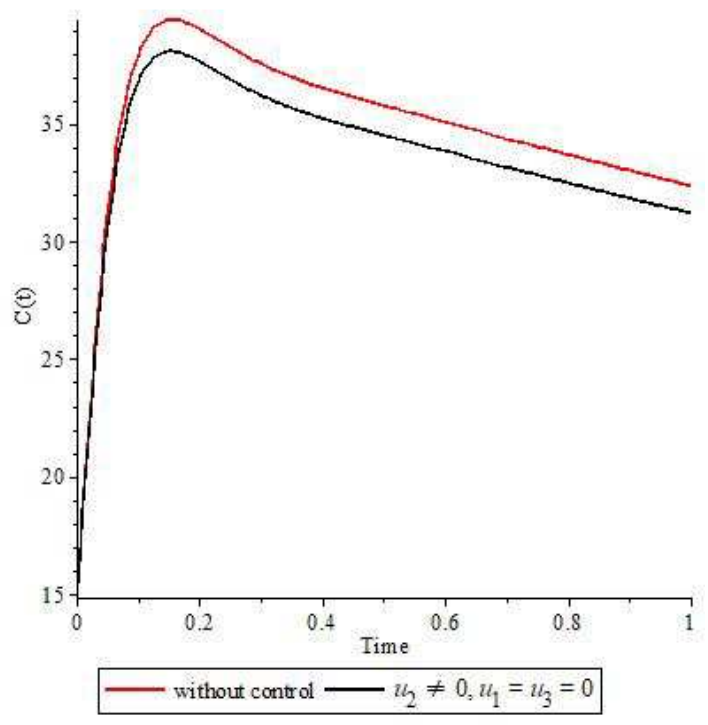

Figure 19. Compartment $C(t)$ with one controls, $u_{2} \neq 0$.

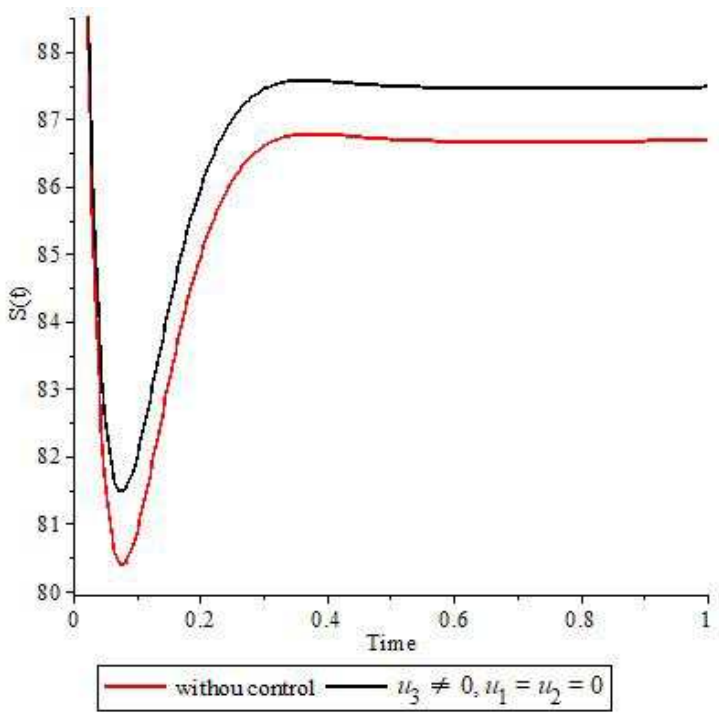

Figure 20. Compartment $S(t)$ with one controls, $u_{2} \neq 0$

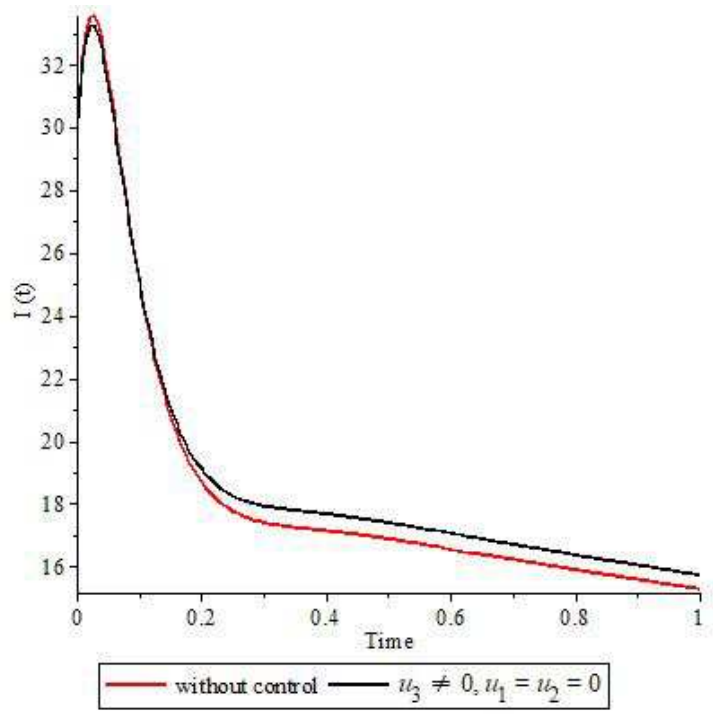

Figure 21. Compartment I $(t)$ with one controls, $u_{2} \neq 0$. 


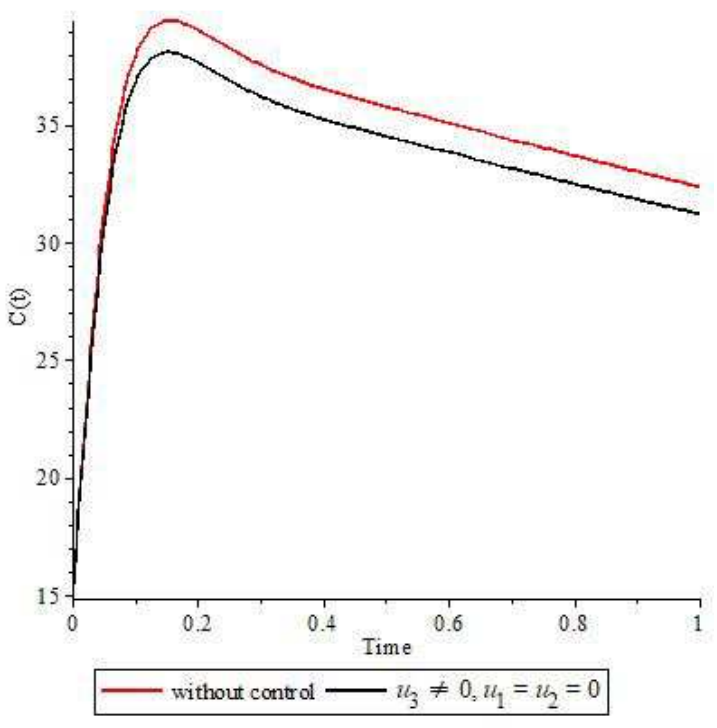

Figure 22. Compartment $C(t)$ with one controls, $u_{2} \neq 0$

\section{Discussion}

Obviously, from Figure 2, without control measures $S(t)$ compartment reduces until an equilibrium position is obtained due to the fact that to meet up with the daily obligation from work, they neglect engaging in profitable physical activities that can lower the risk of $C V D$. Hence, more people emigrate from $S(t)$ compartment to $I(t)$ and subsequently to $C(t)$ as time goes on. With the control measures in place, more people tends to understand the need for such exercises and hence the compartment increases.

Similarly, from Figure 3, without control the compartment increases which later reduces as people started to develop $C V D$, while with control, downward trend is recorded. From Figure 4, without control, there is a risk of spike in CVD related to sedentary lifestyle as obtained from the graph while with control, cases of $C V D$ reduces even among the ones that have it as a result of hereditary.

In the following graphs, we want to test the efficacy of two of the controls while setting the remaining one to zero. From Figures $5-13$, it can be observed that, the combination of control one with any other control gives a better result than any other combinations, hence it highlighted the impact of proper education to reduce the risk of new cases of $C V D$ from sedentary lifestyle.

In the next graphs, we want to set two control parameters to zero in order to know the control that have the highest impact from the model. Obviously, control $u_{1} \neq 0$ prove to be the control that yields a faster result. This is in no wise saying that other controls are not useful, but its reiterating the fact that so many people are quiet aware of importance of nutrition, weight management and others, but the correlation between a sedentary lifestyle and $C V D$ needs to be clarified to people. Hence, they need to be properly educated on the need to maintain a lifestyle of profitable physical exercise in order to reduce the risk of developing the $C V D$.

\section{Conclusion}

In this paper, a nonlinear deterministic model for optimal control of sedentary lifestyle in relation to cardiovascular disease is developed and analyzed. Some of the findings in the study are:

i. the model investigate the links between sedentary behavior and the disease development and the result obtain shows that, if the sedentary behavior continues over a prolonged period evidence suggests it could transition to a pathological condition that leads to $C V D$ disease since chronic disease can take years to develop.

ii. The model basic properties for Positivity and the Boundedness of solution are tested and were found to exist, non-negative for all $t \geq 0$ with non-negative initial conditions in, $R_{3}^{+}$.

iii. The model was extended and optimal control problem is designed by applying Pontryagin Maximum Principle with three control strategies such like: education or sensitization, living a healthy lifestyles and plenty physical activity. Furthermore, the impact of using possible combinations of the three control interventions were investigated and analyzed.

iv. Numerical simulations of the extended model shows that combination of control $u_{1}$ with any other control gives better result than any other combinations. Therefore, it was assume to be the most suitable and effective strategy to reduce the risk of new cases of $C V D$ from sedentary lifestyle.

\section{References}

[1] ADAPTS (2019).

6-strategies-for-reducing-the-risk-of-prolonged-sitting-at-wor k, URL:

https://www.adaptergonomics.com/canada/6-strategies-for-red ucing-the-risk-of-prolonged-sitting-at-work/assessed.

[2] A. H. A. (2019). American Heart Association. URL: https://Www. Heart.

Org/En/Health-Topics/Consumer-Healthcare/What-Is-Cardiov ascular-Disease/Family-History-And-Heart-Disease-Stroke.

[3] V. Bindu, K. R. Padma, C. Madhuri, D. Sarada (2019). A Study On Coronary Heart Disease Prevention-Nutritional Status, Dietary Patterns and Life Stlye Management, International Journal of Scientific \& Technology Research 8 (11).

[4] D. Dunstan, G. Healy, N. Owen, J. Salmon, N. Ridgers, T. Okely, D. Cliff, M. Daley, A. Bauman (2019). Prolonged sitting. In: Blueprint for an Active Australia. 3rd ed. Melbourne: National Heart Foundation of Australia.

[5] HRIASIA (2019).

Far-Reaching-Business-Implications-Of-A-Sedentary-Workfo rce-Do-You-Encourage-Prolonged-Sitting https://www.hrinasia.com/people-development/far-reaching-b usiness-implications-of-a-sedentary-workforce-do-you-encour age-prolonged-sitting/.

[6] M. O. Ibrahim, S. T. Akinyemi, M. M. Dogo, and N. G Bakare (2015). Mathematical Modeling of a Stage Progression HIV/ AIDS Model with Control Measures, Journal of Nigerian Ass of Math Physics, vol 9 151-162. 
[7] L. Jibril and A. U. Moyi (2019). Differential Transform Method for the Vaccination Model of the cholera Carrier, International Research Journal of Engineering and Technology (IRJET) 6 (11) 2379-2386.

[8] M. D. W. Kraus (2015). Family History and Heart Disease, Stroke, American Heart Association.

https://www.heart.org/en/health-topics/consumer-healthcare/what-is -cardiovascular-disease/family-history-and-heart-disease-stroke.

[9] S. Lenhart and J. T. Workman (2007). Optimal Control applied to biological Models. United States of America: Chapman \& Hall/CRC.

[10] M. D. Michos (2015, July 17). Health. Retrieved from John Hopkins Medicine: URL:

https://www.hopkinsmedicine.org/health/wellness-and-preven tion/sitting-disease-how-a-sedentary-lifestyle-affects-heart-he alth.

[11] N. H. S (2018). URL: https://Www.nhs. Uk/Conditions/Cardiovascular-Disease/.

[12] L. S. Pontryagin, V. G. Boltyanskii, R. V. Gamkrelize and E. F. Mischenko (1962). The mathematical theory of optimal processes. New York: Wiley.

[13] D. Shambhavi and K. Bansal (2019). A study of cardiovascular risk behaviour among medical students in a medical college, Gurugram Haryana", International Journal of Current Research, $11,(11), 8451-8453$.

[14] J. P. Thyfault, D. Mengmeng, W. E Kraus, J. A. Levine, and F. W. Booth (2015). Physiology of Sedentary Behavior and Its Relationship to Health Outcomes, Med Sci Sports Exerc, 47 (6): 1301-1305.

[15] UTKATA (2019).

What-Sitting-all-Day-does-toyour-Body-and-how-to-Reverset he-Harmful-Effects, URL:

https://utkatayoga.com/what-sitting-all-day-does-to-your-body -and-how-to-reverse-the-harmful-effects/.

[16] Wikipedia (2014). Sedentary Life styes, URL:

https://www.lifespanfitness.cm/workplace/resources/articles/si tting-all-day-is-taking-a-toll- on-your-body.

[17] World Health Organisation.

URL:https://www.who.int/news-room/fact-sheets/detail/cardio vascular-diseases-(cvds).

[18] W. O. Kermack, A. G. McKendrick, A contribution to the mathematical theory of epidemics, Proceedings of the Royal Society of London. Series A, Containing Papers of a Mathematical and Physical Character 115 (1927) 700-721. 